\title{
A NEW ANALYTICAL JET MODEL
}

\author{
C.R. KAISER AND P. ALEXANDER \\ MRAO, Cavendish Lab., Madingley Road, Cambridge, UK.
}

\begin{abstract}
We present a self-consistent, self-similar model for classical radio double sources (FRIIs). This model depends only on quantities which can in principle be measured.
\end{abstract}

\section{Motivation and assumptions}

Since the standard model for FRIIs was introduced by Scheuer (1974) observations and numerical simulations have provided us with new insights into the properties and mechanisms of these radio sources. In our model we are using some of these results to form our basic assumptions.

First, we note from observations of FRIIs that the ratio of width to length of sources on very different scales are similar (e.g. Black 1992). This suggests that sources are growing in a self-similar way. Therefore we adopt for the volume of the cocoon $V \propto L^{3}$, where $L$ is the length of the source.

Numerical simulations have shown that the jet itself can be confined by the pressure in the cocoon which is inflated by the shocked jet material. We assume a jet with no opening angle but constant cross section in pressure balance with the cocoon material. This assumption was also made by Begelman et al.(1989) and verified with numerical simulations for their model by Cioffi et al. (1992). Magnetic fields do not play a role in confining the jet and are taken to be dynamically unimportant in the cocoon in the sense that they add to the total pressure, but do not exert a non-isotropic stress, i.e. magnetic field lines are tangled on a small scale.

Assuming a strong, non-radiative shock we find for a relativistic gas in the cocoon $(\Gamma=4 / 3)$ the sound speed to be about 0.4 times the velocity of the material in the jet. Since this material is relativistic the sound speed in the cocoon is very high and differences in the pressure in different parts of the cocoon will smooth out quickly. Therefore we assume a uniform pressure within the cocoon. 


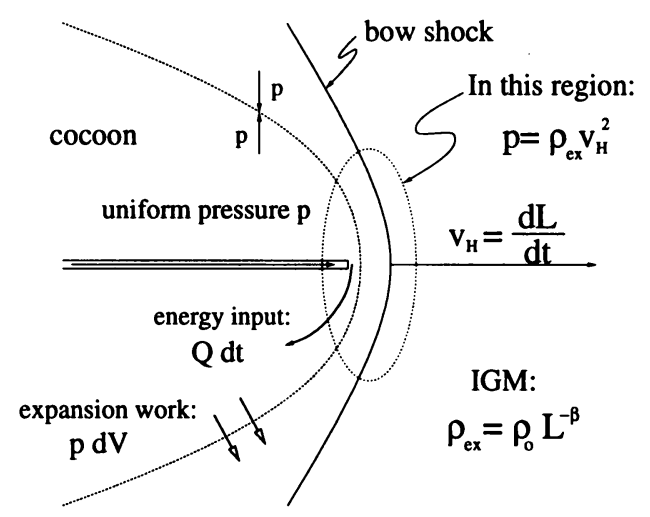

Figure 1. Basic model setup

\section{Model dynamics}

Fig. 1 shows the basic setup of the model. Close to the hotspot we balance the pressure in the cocoon with the ram pressure of the IGM. That means that the whole front of the cocoon has to push through the ambient medium and not just the rather small area where the jet hits the surrounding gas. In order to conserve energy we must have:

$$
Q d t=\frac{\Gamma}{\Gamma-1} p d V+V d p
$$

The jet power $Q$ is assumed to be constant. With this, the expression for the density of the surrounding gas given in fig.1, and the assumption made in section 1 we can derive:

$$
L \propto t^{\frac{3}{5-\beta}}, p \propto t^{\frac{-4-\beta}{5-\beta}}
$$

The constants of proportionality in both cases depend only on the jet power, the constants $\rho_{o}$ and $\beta$ for the external medium, and the ratio of cocoon width to length. All of these can be determined from observations (for $Q$ see Rawlings et al. 1991).

\section{References}

Begelmann, M.C. and Cioffi, D.F. 1989, ApJ, 345, L21. Black, A.R.S. 1992, Ph.D. thesis, University of Cambridge.

Cioff, D.F. and Blondin, J.M. 1992, $A p J, 392,458$.

Rawlings, S. and Saunders, R. 1991, Nature, 349, 138.

Scheuer, P.A.G. 1974, M.N.R.A.S., 166, 513. 